\title{
plasp: A Prototype for PDDL-Based Planning in ASP
}

\author{
Martin Gebser, Roland Kaminski, Murat Knecht, and Torsten Schaub* \\ Institut für Informatik, Universität Potsdam
}

\begin{abstract}
We present a prototypical system, plasp, implementing Planning by compilation to Answer Set Programming (ASP). Our approach is inspired by Planning as Satisfiability, yet it aims at keeping the actual compilation simple in favor of modeling planning techniques by meta-programming in ASP. This has several advantages. First, ASP modelings are easily modifiable and can be studied in a transparent setting. Second, we can take advantage of available ASP grounders to obtain propositional representations. Third, we can harness ASP solvers providing incremental solving mechanisms. Finally, the ASP community gains access to a wide range of planning problems, and the planning community benefits from the knowledge representation and reasoning capacities of ASP.
\end{abstract}

\section{Introduction}

Boolean Satisfiability (SAT; [1]) checking provides a major implementation technique for Automated Planning [2]. In fact, a lot of efforts have been made to develop compilations mapping planning problems to propositional formulas. However, the underlying techniques are usually hard-wired within the compilers, so that further combinations and experiments with different features are hard to implement.

We address this situation and propose a more elaboration-tolerant platform to Planning by using Answer Set Programming (ASP; [3]) rather than SAT as target formalism. The idea is to keep the actual compilation small and model as many techniques as possible in ASP. This approach has several advantages. First, planning techniques modeled in ASP are easily modifiable and can be studied in a transparent setting. Second, we can utilize available ASP grounders to obtain propositional representations. Third, we can harness ASP solvers providing incremental solving mechanisms. Finally, the ASP community gains access to a wide range of planning problems, and the planning community benefits from the knowledge representation and reasoning capacities of ASP.

Our prototypical system, plasp, follows the approach of SATPlan [4,5] in translating a planning problem from the Planning Domain Definition Language (PDDL; [6]) into Boolean constraints. Unlike SATPlan, however, we aim at keeping the actual compilation simple in favor of modeling planning techniques by meta-programming in ASP. Although the compilations and meta-programs made available by plasp do not yet match the sophisticated approaches of dedicated planning systems, they allow for applying ASP systems to available planning problems. In particular, we make use of the incremental ASP system iClingo [7], supporting the step-wise unrolling of problem horizons. Our case studies demonstrate the impact of alternative compilations and ASP modelings on the performance of $i$ Clingo.

\footnotetext{
* Affiliated with Simon Fraser University, Canada, and Griffith University, Australia.
} 


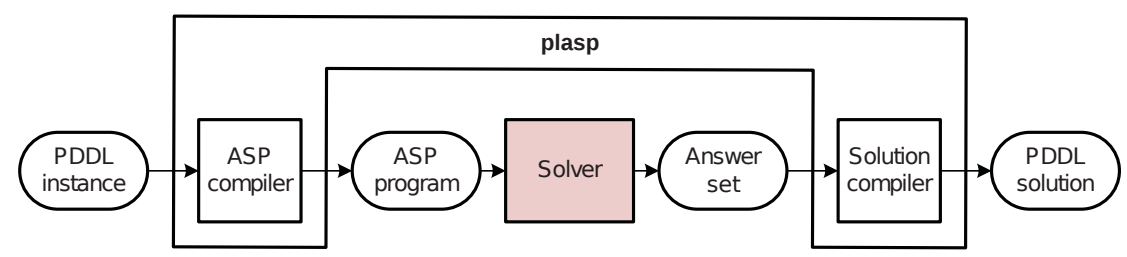

Fig. 1. Architecture of the plasp system.

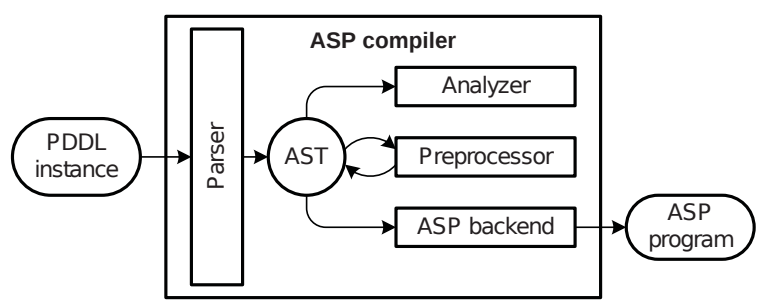

Fig. 2. Architecture of the ASP compiler.

\section{Architecture}

As illustrated in Figure 1, plasp translates a PDDL problem instance to ASP and runs it through a solver producing answer sets. The latter represent solutions to the initial planning problem. To this end, a plan is extracted from an answer set and output in PDDL syntax. plasp thus consists of two modules, viz., the ASP and Solution compilers. The ASP compiler is illustrated in Figure 2. First, a parser reads the PDDL description as input and builds an internal representation, also known as Abstract Syntax Tree (AST). Then, the Analyzer gathers information on the particular problem instance; e.g., it determines predicates representing fluents. Afterwards, the Preprocessor modifies the instance and enhances it for the translation process. Finally, the ASP backend produces an ASP program using the data gathered before. The Solution compiler constructs a plan from an answer set output by the solver. This is usually just a syntactic matter, but it becomes more involved in the case of parallel planning where an order among the actions must be re-established. Afterwards, the plan is verified and output in PDDL syntax.

\section{Compilations and Meta-Programs}

In order to give an idea of the resulting ASP programs, let us sketch the most basic planning encoding relying on meta-programming. To this end, a PDDL domain description is mapped onto a set of facts built from predicates init, goal, action, demands, adds, and deletes along with their obvious meanings. Such facts are then combined with the meta-program in Figure 3. Note that this meta-program is treated incrementally by the ASP system iClingo, as indicated in lines (1), (3), and (10). While the facts resulting from the initial PDDL description along with the ground rules of (2) are processed just once, the rules in (4)-(9) are successively grounded for increasing values of $t$ and accumulated in iClingo's solving component. Finally, goal conditions are expressed by 
(1) \#base.

(2) $\operatorname{holds}(F, 0) \leftarrow \operatorname{init}(F)$.

(3) \#cumulative $t$.

(4) $1\{\operatorname{apply}(A, t): \operatorname{action}(A)\} 1$.

$(5) \leftarrow \operatorname{apply}(A, t)$, demands $(A, F$, true $), \operatorname{not} h o l d s(F, t-1)$.

$(6) \leftarrow \operatorname{apply}(A, t)$, demands $(A, F$, false $)$, holds $(F, t-1)$.

(7) $\operatorname{holds}(F, t) \leftarrow \operatorname{apply}(A, t), \operatorname{adds}(A, F)$.

(8) $\operatorname{del}(F, t) \leftarrow \operatorname{apply}(A, t), \operatorname{deletes}(A, F)$.

(9) $\operatorname{holds}(F, t) \leftarrow \operatorname{holds}(F, t-1), \operatorname{not} \operatorname{del}(F, t)$.

(10) \#volatile $t$.

(11) $\leftarrow \operatorname{goal}(F$, true $)$, not holds $(F, t)$.

$(12) \leftarrow \operatorname{goal}(F$, false $), \operatorname{holds}(F, t)$.

Fig. 3. Basic ASP encoding of STRIPS planning.

$\left(4^{\prime} a\right) \leftarrow \operatorname{apply}\left(A_{1}, t\right), \operatorname{apply}\left(A_{2}, t\right), A_{1} \neq A_{2}, \operatorname{demands}\left(A_{1}, F\right.$, true $)$, deletes $\left(A_{2}, F\right)$.

$\left(4^{\prime} b\right) \leftarrow \operatorname{apply}\left(A_{1}, t\right), \operatorname{apply}\left(A_{2}, t\right), A_{1} \neq A_{2}$, demands $\left(A_{1}, F\right.$, false $)$, adds $\left(A_{2}, F\right)$.

$\left(4^{\prime} c\right) \leftarrow \operatorname{apply}\left(A_{1}, t\right), \operatorname{apply}\left(A_{2}, t\right), A_{1} \neq A_{2}, \operatorname{adds}\left(A_{1}, F\right)$, deletes $\left(A_{2}, F\right)$.

Fig. 4. Adaptation of the basic ASP encoding to parallel STRIPS planning.

volatile rules, contributing ground rules of (11) and (12) only for the current step $t$. See [7] for further details on incremental ASP solving. From a representational perspective, it is interesting to observe that ASP allows for omitting a frame axiom (like the one in line (9)) for negative information, making use of the fact that instances of holds are false by default, that is, unless they are explicitly derived to be true. Otherwise, the specification follows closely the semantics of STRIPS [2].

Beyond the meta-program in Figure 3, plasp offers planning with concurrent actions. The corresponding modification of the rule in $(4)$ is shown in Figure 4. While $\left(4^{\prime}\right)$ drops the uniqueness condition on applied actions, the additional integrity constraints stipulate that concurrent actions must not undo their preconditions, nor have conflicting effects. The resulting meta-program complies with the $\forall$-step semantics in [8]. Furthermore, plasp offers operator splitting as well as forward expansion. The goal of operator splitting [9] is to reduce the number of propositions in the representation of a planning problem by decomposing action predicates; e.g., an action $a(X, Y, Z)$ can be represented in terms of $a_{1}(X), a_{2}(Y), a_{3}(Z)$. Forward expansion (without mutex analysis [10]) instantiates schematic actions by need, viz., if their preconditions have been determined as feasible at a time step, instead of referring to statically given instances of the action predicate. This can be useful if initially many instances of a schematic action are inapplicable, yet it requires a domain-specific compilation; meta-programming is difficult to apply because action instances are not represented as facts. Finally, plasp supports combinations of forward expansion with either concurrent actions or operator splitting. Regardless of whether forward expansion is used, concurrent actions and operator splitting can currently not be combined; generally, both techniques are in opposition, although possible solutions have recently been proposed [11]. 


\begin{tabular}{|l||r|r|r|r|r|r||r||r|}
\hline Benchmark & basic & concur & split & expand & concur+expand & split+expand & SATPlan & SGPlan \\
\hline \hline Blocks-4-0 & 0.16 & 0.21 & 0.43 & 0.21 & 0.22 & 0.20 & 0.34 & 0.10 \\
Blocks-6-0 & 0.30 & 0.63 & 0.93 & 0.44 & 0.56 & 1.40 & 0.27 & 0.04 \\
Blocks-8-0 & 1.58 & 6.53 & 12.78 & 98.60 & 317.53 & 47.57 & 1.24 & 0.09 \\
\hline Elevator-3-0 & 0.27 & 0.56 & 0.92 & 0.30 & 0.46 & 0.89 & 0.10 & 0.02 \\
Elevator-4-0 & 11.72 & 264.11 & 20.30 & 14.69 & 324.18 & 28.88 & 0.30 & 0.02 \\
Elevator-5-0 & - & - & 320.58 & - & - & 467.98 & 0.61 & 0.04 \\
\hline FreeCell-2-1 & 93.42 & mem & 64.28 & 60.52 & 51.33 & 56.94 & 2.44 & 0.12 \\
FreeCell-3-1 & - & mem & - & - & 175.03 & - & 10.44 & 0.14 \\
\hline Logistics-4-0 & 7.85 & 0.38 & 79.15 & 8.81 & 0.39 & 70.56 & 0.34 & 0.05 \\
Logistics-7-0 & - & 0.99 & - & - & 0.61 & - & 0.31 & 0.04 \\
Logistics-9-0 & - & 0.89 & - & - & 0.57 & - & 0.27 & 0.04 \\
\hline Satellite-1 & 0.23 & 0.87 & 0.23 & 0.29 & 0.74 & 0.26 & 0.10 & 0.03 \\
Satellite-2 & 4.56 & 638.08 & 2.19 & 5.43 & 448.60 & 2.69 & 0.41 & 0.03 \\
Satellite-3 & 8.76 & 3.52 & 4.00 & 7.54 & 3.29 & 3.70 & 0.21 & 0.04 \\
\hline Schedule-2-0 & mem & mem & mem & 1.03 & 3.37 & mem & mem & mem \\
Schedule-3-0 & mem & mem & mem & 1.63 & 12.89 & mem & mem & mem \\
\hline
\end{tabular}

Table 1. Experiments comparing different compilations.

\section{Experiments}

We conducted experiments comparing the different compilation techniques furnished by plasp $^{1}$ (1.0): the meta-program in Figure 3 (column "basic" in Table 1), its adaptation to concurrent actions in Figure 4 ("concur"), operator splitting ("split"), forward expansion ("expand"), and two combinations thereof ("concur+expand" and "split+expand"). To compute answer sets of compilations, representing shortest plans, plasp uses (a modified version of) the incremental ASP system $i_{\text {Clingo }}{ }^{1}$ (2.0.5). Although we mainly study the effect of different compilations on the performance of iClingo, for comparison, we also include SATPlan ${ }^{2}$ (2006) and SGPlan ${ }^{3}$ (5.2.2). While SGPlan [12] does not guarantee shortest plan lengths, the approach of SATPlan, based on compilation and the use of a SAT solver as search backend, leads to shortest plans. In fact, its compilation is closely related to the "concur+expand" setting of plasp, where SATPlan in addition applies mutex analysis. The benchmarks, formulated in the STRIPS subset ${ }^{4}$ of PDDL, stem from the Second International Planning Competition ${ }^{4}$, except for the three Satellite instances taken from the fourth competition ${ }^{5}$. All experiments were run on a Linux PC equipped with $2 \mathrm{GHz}$ CPU and 2 GB RAM, imposing 900 seconds as time and $1.5 \mathrm{~GB}$ as memory limit.

Runtime results in seconds are shown in Table 1; an entry "_-" indicates a timeout, and "mem" stands for memory exhaustion. On all benchmarks but Schedule, we observe that SGPlan has an edge on the other, less specialized (yet guaranteeing shortest

\footnotetext{
${ }^{1}$ http://potassco.sourceforge. net

${ }^{2}$ http: //www.cs.rochester. edu/ kautz/satplan

${ }^{3}$ http://manip.crhc.uiuc.edu/programs/SGPlan

${ }^{4}$ http: //www.cs.toronto.edu/aips 2000

5 http://www.tzi.de/ edelkamp/ipc-4
} 
plans) systems. The fact that SATPlan is usually faster than plasp can be explained by the fact that compilations of plasp are instantiated by a general-purpose ASP grounder, while SATPlan utilizes a planning-specific frontend [10]. Moreover, mutex analysis as in SATPlan is currently not included in (encodings of) plasp. However, we observe that different compilation techniques of plasp pay off on particular benchmarks. On the Blocks and small Elevator instances, the simplest meta-program ("basic") is superior because concurrency and expansion are barely applicable to them and may even deteriorate performance. On Elevator-5-0, splitting ("split") helps to reduce the size of the problem representation. Furthermore, we observe that allowing for concurrent actions without explicit mutexes ("concur" and "concur+expand") dramatically decreases search efficiency on the Elevator domain. However, concurrent actions in combination with forward expansion ("concur+expand") are valuable on FreeCell and Logistics instances, given that they involve non-interfering actions. Splitting ("split" and "split+expand") appears to be useful on Satellite instances, where Satellite-2 again yields the phenomenon of concurrent actions deteriorating search. Finally, forward expansion ("expand") enables plasp to successfully deal with the Schedule domain, where even SATPlan and SGPlan exceed the memory limit. We conjecture that (too) exhaustive preprocessing, e.g., mutex analysis, could be responsible for this.

In summary, we conclude that the different compilation techniques of plasp can be advantageous. The automatic, domain-specific choice of an appropriate compilation, required in view of varying characteristics [12], is an intrinsic subject to future work.

\section{Discussion}

We have presented a prototypical approach to Automated Planning by means of compilation to ASP. In order to close the gap to established planning systems, more background knowledge (e.g., mutexes) would need to be included. If such knowledge can be encoded in meta-programs, it fosters elaboration tolerance and flexibility of planning implementations. In fact, the recent version transition of iClingo from 2 to 3 gives inherent support of forward expansion, generating the possibly applicable instances of actions (and fluents) on-the-fly during grounding. Importantly, regardless of additional features that might boost performance (cf. [13]), the compilation capacities of plasp are already useful as they make various planning problems, formulated in PDDL, accessible as benchmarks for ASP systems. The range could be further extended by generalizing the compilations supported by plasp beyond the STRIPS subset of PDDL.

Given the proximity of Planning and General Game Playing (GGP; [14]), the latter can also (partially) be implemented by compilation to ASP. An approach to solve singleplayer games in ASP is provided in [15], and [16] presents ASP-based methods to prove properties of games, which can then be exploited for playing. Automatically proving properties of interest to steer the selection of solving techniques may also be useful for Planning. Another line of future work could be Conformant Planning [17], whose elevated complexity could be addressed by compilation to disjunctive ASP. In fact, the $d l v^{\mathcal{K}}$ system [18] supports Conformant Planning wrt action language $\mathcal{K}$.

Acknowledgments. This work was partly funded by DFG grant SCHA 550/8-2. 


\section{References}

1. Biere, A., Heule, M., van Maaren, H., Walsh, T.: Handbook of Satisfiability. IOS Press (2009)

2. Nau, D., Ghallab, M., Traverso, P.: Automated Planning: Theory and Practice. Morgan Kaufmann (2004)

3. Baral, C.: Knowledge Representation, Reasoning and Declarative Problem Solving. Cambridge University Press (2003)

4. Kautz, H., Selman, B.: Planning as satisfiability. Proceedings of the Tenth European Conference on Artificial Intelligence (ECAI'92), Wiley (1992) 359-363

5. Kautz, H., Selman, B.: Pushing the envelope: Planning, propositional logic, and stochastic search. Proceedings of the Thirteenth National Conference on Artificial Intelligence (AAAI'96), AAAI/MIT Press (1996) 1194-1201

6. McDermott, D.: PDDL - the planning domain definition language. Technical Report CVC TR-98-003/DCS TR-1165, Yale Center for Computational Vision and Control (1998)

7. Gebser, M., Kaminski, R., Kaufmann, B., Ostrowski, M., Schaub, T., Thiele, S.: Engineering an incremental ASP solver. Proceedings of the Twenty-fourth International Conference on Logic Programming (ICLP'08), Springer (2008) 190-205

8. Rintanen, J., Heljanko, K., Niemelä, I.: Parallel encodings of classical planning as satisfiability. Proceedings of the Ninth European Conference on Logics in Artificial Intelligence (JELIA'04), Springer (2004) 307-319

9. Kautz, H., McAllester, D., Selman, B.: Encoding plans in propositional logic. Proceedings of the Fifth International Conference on Principles of Knowledge Representation and Reasoning (KR'96), Morgan Kaufmann (1996) 374-384

10. Blum, A., Furst, M.: Fast planning through planning graph analysis. Artificial Intelligence 90(1-2) (1997) 279-298

11. Robinson, N., Gretton, C., Pham, D., Sattar, A.: SAT-based parallel planning using a split representation of actions. Proceedings of the Nineteenth International Conference on Automated Planning and Scheduling (ICAPS'09), AAAI Press (2009) 281-288

12. Hsu, C., Wah, B., Huang, R., Chen, Y.: Constraint partitioning for solving planning problems with trajectory constraints and goal preferences. Proceedings of the Twentieth International Joint Conference on Artificial Intelligence (IJCAI'07), AAAI/MIT Press (2007) 1924-1929

13. Sideris, A., Dimopoulos, Y.: Constraint propagation in propositional planning. Proceedings of the Twentieth International Conference on Automated Planning and Scheduling (ICAPS'10), AAAI Press (2010) 153-160

14. Genesereth, M., Love, N., Pell, B.: General game playing: Overview of the AAAI competition. AI Magazine 26(2) (2005) 62-72

15. Thielscher, M.: Answer set programming for single-player games in general game playing. Proceedings of the Twenty-fifth International Conference on Logic Programming (ICLP'09), Springer (2009) 327-341

16. Thielscher, M., Voigt, S.: A temporal proof system for general game playing. Proceedings of the Twenty-fourth National Conference on Artificial Intelligence (AAAI'10), AAAI Press (2010) 1000-1005

17. Smith, D., Weld, D.: Conformant Graphplan. In: Proceedings of the Fifteenth National Conference on Artificial Intelligence (AAAI'98), AAAI/MIT Press (1998) 889-896

18. Eiter, T., Faber, W., Leone, N., Pfeifer, G., Polleres, A.: A logic programming approach to knowledge-state planning. Artificial Intelligence 144(1-2) (2003) 157-211 